\title{
Aminotransferasas séricas en pacientes con Dengue tipo 3
}

\author{
Nereida Valero $\mathbf{C}^{\mathbf{a}}$, Ivette Reyes $\mathbf{V}^{\mathbf{b}}$, Yraima Larreal $\mathrm{E}^{\mathrm{c}}$, \\ Mery Maldonado $\mathrm{E}^{\mathrm{a}}$.
}

\section{Aminotransferases serum levels in patients with Dengue type 3}

Background: Dengue infections may affect the liver, causing inflammation and compromising its function. Aim: To determine serum aminotransferases in patients with Dengue. Material and methods: One hundred eighty four patients with Dengue confirmed with anti-Dengue IgG and IgM antibodies measured by ELISA, aged 1 month to 79 years, were studied. As controls, 40 patients with acute hepatitis B virus infection aged 11 to 59 years and 40 healthy individuals, aged 14 to 55 years, were also studied. Alanine and aspartate aminotransferases (ALT and AST) were determined using a colorimetric method. Results: Sixty one percent of patients with Dengue had elevated aminotransferase levels. In patients with Dengue and hepatitis B, mean ALT levels were $21.3 \pm 8.4$ and $29.7 \pm 18.8 \mathrm{U} / \mathrm{I}$, respectively. Mean AST values were $37.3 \pm 19,19.6 \pm 16.4$ and $4.3 \pm 1.7 \mathrm{U} / \mathrm{I}$ in patients with Dengue, hepatitis B and controls, respectively. During the year 2001, there was an increase in the number of cases with hemorrhagic fever caused by Dengue and $65 \%$ had elevation of aminotransferases. Conclusions: An increase in aminotransferase levels is common in patients with Dengue (Rev Méd Chile 2007; 135: 1304-12).

(Key words: Dengue hemorrhagic fever; Hepatitis, viral, human; Transaminases)

Recibido el 13 de enero, 2006. Aceptado el 7 de noviembre, 2006.

Sección de Virología, Instituto de Investigaciones Clínicas "Dr. Américo Negrette”, Facultad de Medicina, Universidad del Zulia, Maracaibo-Estado Zulia, Venezuela

aMgSc en Biología, Inmunología Básica

bLicenciada en Bioanálisis

${ }^{\mathrm{c} D o c t o r a}$ en Ciencias Médicas, especialista en Medicina Interna

Las as infecciones por dengue provocan un espectro de presentaciones que varía desde un proceso asintomático, fiebre indiferenciada o fiebre por dengue (FD) hasta fiebre hemorrágica por

Correspondencia a: MgSc Nereida Valero Cedeño. Facultad de Medicina, Universidad del Zulia, Maracaibo-Estado Zulia, Venezuela. Fono 58-261-7597294. Fax: 58-2617597247; 0414-3624181. Apartado postal 23.

E mail: nere98@hotmail.com. dengue (FHD) y evolucionar a síndrome de shock (SSD), presentándose con frecuencia manifestaciones sistémicas, debido al marcado tropismo del virus por los órganos del sistema monocíticomacrofágico, como son la médula ósea, bazo, nódulos linfáticos e hígado ${ }^{1,2}$. En este caso, el dengue puede producir afección en el hígado, caracterizada por inflamación y reducción de su capacidad funcional, pudiendo dar lugar a resultados anormales en pruebas de funcionalismo hepático. Algunos autores sostienen que el virus 
dengue es hepatotropo, dado que han detectado antígenos del virus dengue en hepatocitos y partículas virales en muestra de biopsia de hígado en pacientes con $\mathrm{FHD}^{3,4}$. El virus dengue puede infectar el hígado y causar hepatitis.

La hepatitis viral aguda no es una entidad única, sino que puede ser producida por diferentes agentes, entre los que destacan los virus de hepatitis convencional y otros como el virus de Epstein-Barr, el de la fiebre amarilla, el citomegalovirus y el virus Herpes tipo I, cuya lesión hepática tiene relevancia clínica. Los virus de la fiebre Lassa, el de la enfermedad de Marburg y el virus Ébola pueden producir lesión hepática grave y cursar también con cuadros clínicos de fiebre hemorrágica ${ }^{5}$.

Diferentes estudios realizados en regiones asiáticas en las que el dengue es hiperendémico, reportan la presencia de daño hepático en la infección por dengue, con incremento significativo de la alanino aminotransferasa (ALT) y la aspartato aminotransferasa (AST) y en el examen físico dolor abdominal, ictericia y hepatomegalia, similar a lo encontrado en otras hepatitis causadas por virus hepatotropo clásicos ${ }^{6}$.

En el continente americano, ha ocurrido un aumento constante de la incidencia de dengue debido al rápido crecimiento de la población, urbanización no planificada, inadecuado suministro de agua, dificultades en la recolección de residuos sólidos, con el consecuente incremento del mosquito vector, aunado a esto, las migraciones y el incremento en los viajes facilitan también la diseminación de los serotipos virales. Se conocen hasta ahora cuatro serotipos (DEN-1, DEN-2, DEN-3, DEN-4), esta situación hace que se incremente la frecuencia de casos con infecciones secundarias y, consecuentemente, el riesgo de desarrollo de FHD. Otro aspecto que se ha considerado en el complejo fenómeno de la FHD, es la mayor asociación de algunos serotipos con el desarrollo de formas severas de la enfermedad, inclusive con la mayor afectación de órganos y sistemas como es el caso de DEN-2 y DEN-37-14.

En Venezuela, y especialmente en la región zuliana, se ha observado un incremento en los últimos años de FHD acompañado con manifestaciones clínicas de disfunción hepática, lo que hace más difícil para el médico realizar un diagnóstico diferencial en el campo de las infecciones de origen viral, subestimando muchos casos por no tener la posibilidad de un diagnóstico serológico confirmatorio. El objetivo principal de este trabajo fue investigar los valores de ALT y AST en pacientes con infección activa por virus dengue y su relación con la presencia o no de manifestaciones clínicas hepáticas. Adicional a esto, comparar los valores obtenidos en las variantes clínicas de dengue (FD y FHD), con el de los pacientes con infección aguda por virus de hepatitis $B$.

\section{PACIENTES Y MÉTODOS}

Población estudiada. El presente estudio se realizó en el período comprendido entre enero de 1999 y diciembre de 2001. La población estudiada fueron pacientes con clínica compatible con dengue que acudieron a la Sección de Virología del Instituto de Investigaciones Clínicas “Dr. Américo Negrette" de la Facultad de Medicina de la Universidad del Zulia, y del Servicio de Medicina Interna del Hospital General del Sur "Dr. Pedro Iturbe", de la ciudad de Maracaibo.

Se seleccionaron 184 pacientes con un rango de edad de 1 mes de nacido a 79 años, de uno u otro sexo ( $54,3 \%$ pacientes masculinos y $45,6 \%$ femeninos) con confirmación serológica de dengue, $128(69,5 \%)$ de ellos con manifestaciones clínicas de afección hepática (hepatomegalia, ictericia, dolor en hipocondrio derecho). Fueron clasificados de acuerdo a edad, sexo, variantes de dengue de acuerdo al tipo de infección, sintomatología y concentraciones de aminotransferasas ALT y AST.

Además, fueron comparados con 40 pacientes con edades entre 11 y 59 años (47,5\% femenino y $52,5 \%$ masculino) con infección aguda por virus de hepatitis B (positivos a HBsAg y de $14 \pm 3$ días de evolución) y con un grupo control constituido por 40 individuos asintomáticos con edades comprendidas entre 14 y 55 años (40\% femenino y $60 \%$ masculino), con serología negativa para dengue y hepatitis $B$.

Se excluyeron los pacientes que refirieron antecedentes de enfermedad hepática, ingesta de medicamentos, alcohol, exposición a tóxicos y síndromes metabólicos. Además, se excluyeron las muestras hemolizadas (ya que los eritrocitos contienen 3 a 5 veces más ALT que el suero) y esto es 
una interferencia en la cuantificación de aminotransferasas.

Los pacientes manifestaron su autorización por escrito para ser incluidos en el estudio y el protocolo del mismo fue aprobado por el Comité de Ética del Instituto de Investigaciones Clínicas de la Facultad de Medicina de la Universidad del Zulia.

Determinaciones hematológicas y serológicas. A cada paciente se le extrajeron $10 \mathrm{ml}$ de sangre por punción venosa. Tres ml fueron colocados en un tubo de vidrio con EDTA para realizar hematología completa, a través de un contador de células automático (Coulter Counting); el resto fue colocado en un tubo de vidrio seco y estéril, el cual fue centrifugado y del suero obtenido se tomó una parte para la determinación de $\operatorname{IgM}$ e IgG específicas al virus dengue, con la técnica inmunoenzimática de fase sólida ELISA de captura, utilizando el estuche comercial desarrollado por Diag Kit c.a. ${ }^{15}$, utilizando antígenos de los cuatro serotipos del virus dengue.

Tipo de infección. El tipo de infección fue determinado mediante los títulos de anticuerpos IgM e IgG específicos para el virus dengue. En infección primaria IgM es positiva e IgG negativa. En infección secundaria $\operatorname{IgM}$ e $\operatorname{IgG}$ son positivas, tomando en cuenta los días de evolución y que algunos pacientes con infección secundaria presentan IgM bajas o no detectables con títulos muy elevados de $\operatorname{IgG}^{16}$.

Aislamiento e identificación de serotipo de dengue. El aislamiento del virus fue realizado mediante el método de Tesh, inoculación de muestra de suero en la línea celular C6/36 Aedes albopictus e identificado por inmunofluorescencia indirecta con anticuerpos monoclonales serotipo-específico ${ }^{17}$.

Medición de aminotransferasas en suero. Se determinó cuantitativamente la actividad de transferasas (ALT y AST) empleando el método colorimétrico de Reitman-Frankel ${ }^{18}$ con el conjugado comencial de Wiener (Wiener Laboratorio, Argentina), el cual considera valores normales hasta $12 \mathrm{U} / \mathrm{I}$ para ambas aminotransferasas. Se calcularon los resultados de la actividad enzimática con curvas de calibración basadas en la absorbancia del cromógeno, usando un espectrofotómetro a $505 \mathrm{~nm}$.
Análisis estadístico. Los resultados obtenidos fueron ordenados y analizados estadísticamente con el programa Graph Pad 3.0, utilizando ANOVA y como post-test Bonferroni, test de t de Student, chi-cuadrado, según fuera el caso. El registro se realizó mediante tablas y gráficos de distribución de frecuencia que expresan las variables estudiadas. El límite de significancia fue de $\mathrm{p}<0,05$.

\section{RESULTADOS}

Se observó que 112 pacientes con dengue (60,86\%) presentaron un incremento de las aminotransferasas séricas y 72 casos $(39,13 \%)$ con dengue los valores fueron normales. Al analizar según el sexo, del total de pacientes con dengue que presentaron incremento en las aminotransferasas, 68,0\% de la población masculina arrojó valores elevados, mientras que en la femenina fue 52,38\% ( $p<0,05)$.

En los pacientes con dengue con aminotransferasas elevadas, la ALT promedio fue $21,3 \pm 8,3 \mathrm{U} /$ $\mathrm{L}$ y en los pacientes con hepatitis $\mathrm{B}$ aguda 29,7 $\pm 18,8 \mathrm{U} / \mathrm{L}(\mathrm{p}<0,01)$. El control sano: $8,4 \pm 2,5$ U/L (Figura 1). En los pacientes que no presentaron valores alterados de aminotransferasas se observaron valores de ALT de 7,6 $\pm 2,0 \mathrm{U} / \mathrm{L}$.

En los pacientes con dengue que presentaron elevación de aminotransferasas, la AST promedio resultó muy elevada: $37,2 \pm 19 \mathrm{U} / \mathrm{L}(\mathrm{p}<0,001)$ con respecto al control sano: $4,3 \pm 1,7 \mathrm{U} / \mathrm{L}$ y a los pacientes con hepatitis B aguda: 19,6 $\pm 16,4 \mathrm{U} / \mathrm{L}$ (Figura 2). En los pacientes que no presentaron valores alterados de aminotransferasas se obtuvo AST $5,3 \pm 1,7 \mathrm{U} / \mathrm{L}$.

De los 184 pacientes seropositivos para dengue, $48(26,08 \%)$ correspondían a FD y $136(73,91 \%)$ a FHD. Al analizar las variantes de dengue por tipo de infección y las concentraciones de aminotransferasas, se obtuvo que en FD 25\% presentaron aminotransferasas elevadas, en infección primaria $12,5 \%$ y por infección secundaria $50 \%$. Mientras que en FHD 73,52\% presentaron aminotransferasas elevadas, en infección primaria con $55,5 \%$ y por infección secundaria 93,75\% (Tabla 1).

La distribución de casos confirmados por grupos de edad con elevación de aminotransferasas evidenció que el grupo etario de 11 a 20 años $(43,47 \%)$ fue el más afectado, presentando $70 \%$ aminotransferasas elevadas (Tabla 2). 


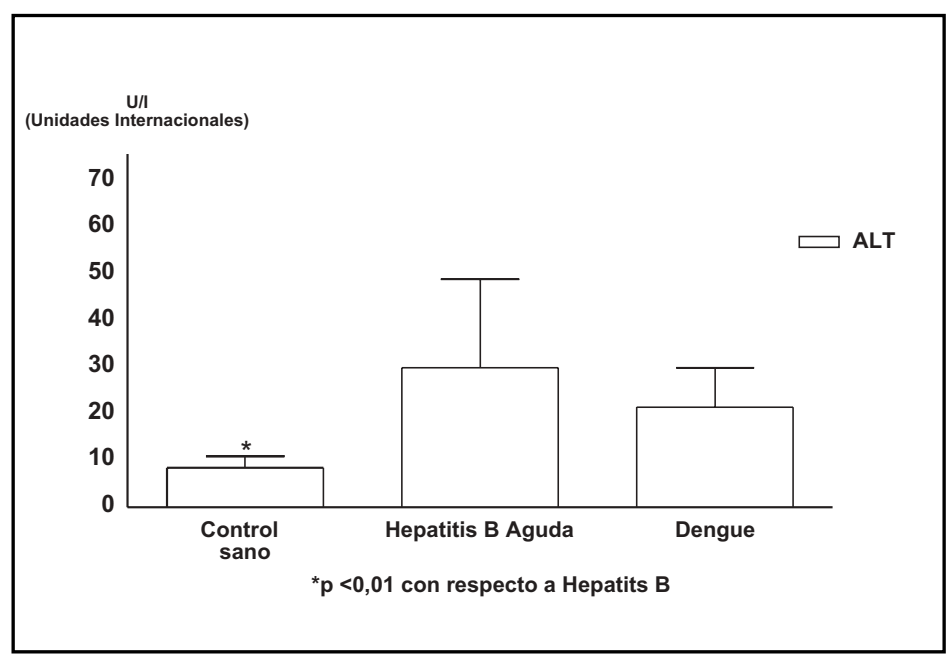

Figura 1. Concentraciones séricas de alaninoaminotransferasa en pacientes con dengue y hepatitis aguda por virus $B$.

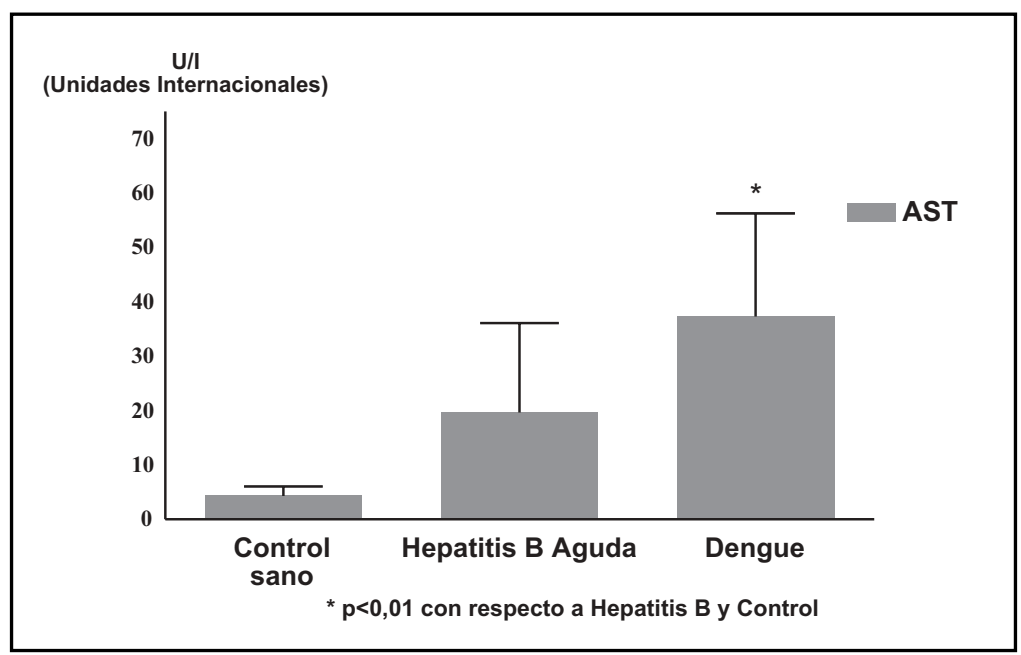

Figura 2. Concentraciones séricas de aspartatoaminotransferasa en pacientes con dengue y hepatitis aguda por virus B.

Tabla 1. Tipo de infección y concentraciones de transferasas en pacientes con dengue en el estado Zulia, Venezuela 1999-2001

\begin{tabular}{|c|c|c|c|c|c|c|c|c|}
\hline \multirow{3}{*}{$\begin{array}{l}\text { Tipo de } \\
\text { Infección }\end{array}$} & \multicolumn{4}{|c|}{ Fiebre por dengue } & \multicolumn{4}{|c|}{ Fiebre hemorrágica por dengue } \\
\hline & \multicolumn{2}{|c|}{ Trans. normales } & \multicolumn{2}{|c|}{ Trans. elevadas } & \multicolumn{2}{|c|}{ Trans. normales } & \multicolumn{2}{|c|}{ Trans. elevadas } \\
\hline & $\mathrm{n}$ & $\%$ & $\mathrm{n}$ & $\%$ & $\mathrm{n}$ & $\%$ & $\mathrm{n}$ & $\%$ \\
\hline Primaria & 28 & 87,5 & 4 & 12,5 & 32 & 44,44 & 40 & 55,5 \\
\hline Secundaria & 8 & 50 & 8 & 50 & 4 & 6,25 & 60 & 93,75 \\
\hline Total & 36 & 75 & 12 & 25 & 36 & 26,4 & 100 & 73,52 \\
\hline
\end{tabular}


Tabla 2. D istribución de casos confirmados de D engue por grupo etario y aminotransferasas elevadas en el Estado Zulia Venezuela, 1999-2001

\begin{tabular}{|cllll|}
\hline Edad (años) & \multicolumn{2}{c}{ Dengue } & \multicolumn{2}{c|}{ Trans. Elevadas } \\
& $\mathrm{n}$ & $\%$ & $\mathrm{n}$ & $\%$ \\
\hline $0-10$ & 24 & 13,04 & 4 & 16,66 \\
$11-20$ & 80 & 43,47 & 56 & 70 \\
$21-30$ & 32 & 17,39 & 24 & 75 \\
$31-40$ & 28 & 15,21 & 16 & 57,14 \\
$>41$ & 20 & 10,86 & 12 & 60 \\
Total & 184 & 100 & 112 & 60,86 \\
\hline
\end{tabular}

Al analizar las concentraciones de ALT en niños y en adultos con dengue se obtuvo que los niños presentaron concentraciones dentro del rango de referencia de ALT $(10 \pm 3,58 \mathrm{U} / \mathrm{L})$. En los adultos se encontraron valores de ALT ligeramente elevados $(17,2 \pm 9,7 \mathrm{U} / \mathrm{L})$; mientras que en los pacientes con hepatitis $B$ aguda se observó un incremento significativo de ALT $(p<0,05)$ con respecto al control sano (Figura 3 ).

Con respecto a las concentraciones de AST en niños y en adultos con dengue se observó que los niños presentaron concentraciones dentro del rango de referencia AST: 9 $\pm 5,6 \mathrm{U} / \mathrm{L}$; mientras que en los adultos se encontraron muy elevados AST:
$28,0 \pm 19,9$ U/L $(p<0,05)$ con respecto al control sano y a los niños con dengue (Figura 4).

$\mathrm{Al}$ analizar la distribución anual de estos pacientes durante el período 1999-2001, se obtuvo que en los años 1999 y 2000, sólo un pequeño porcentaje $(8,33 \%)$ presentaron alteraciones en las concentraciones de aminotransferasas. Mientras que en el año 2001, donde el número de casos fue mayor con predominio de FHD, 64,7\% cursó con elevación en las concentraciones de aminotransferasas; en este mismo año los serotipos circulantes eran DEN-3 (90\% de los casos) y DEN-4 (10\%) (Tabla 3).

$\mathrm{Al}$ relacionar las manifestaciones clínicas de afectación hepática (ictericia, hepatomegalia y

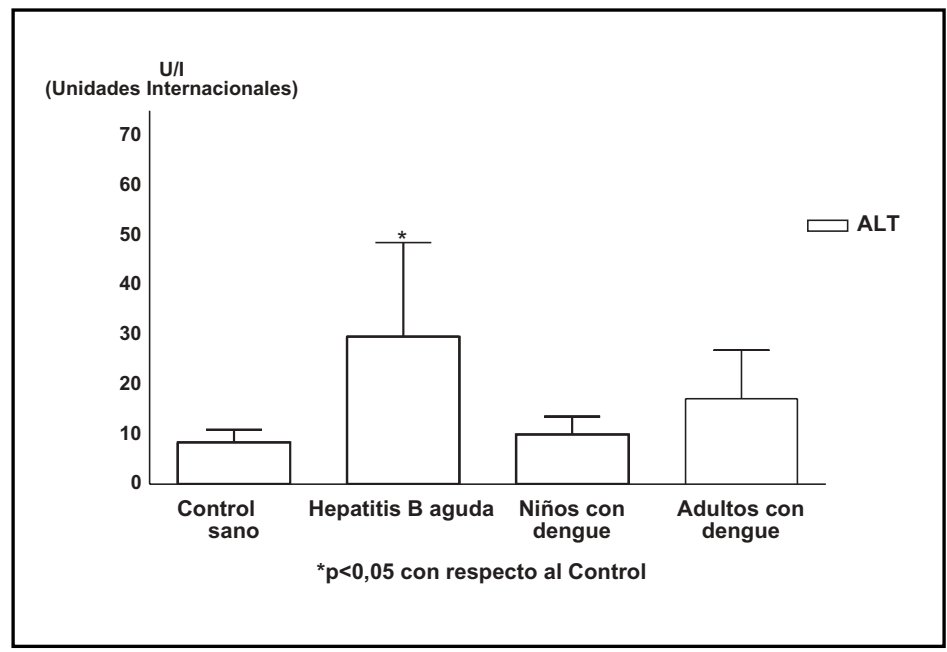

Figura 3. Concentraciones séricas de alaninoaminotransferasas en niños y adultos con dengue. 


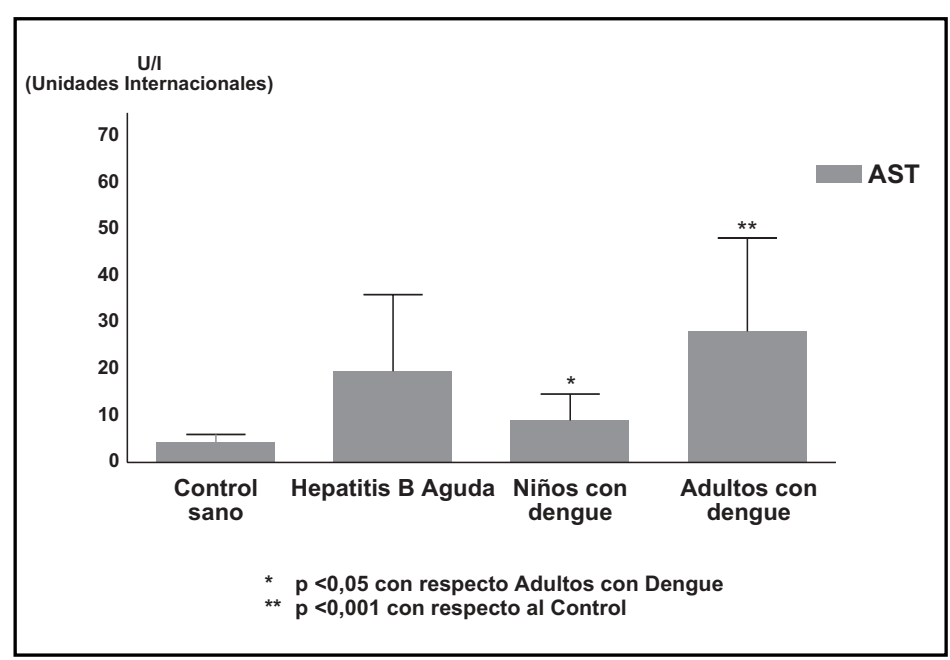

Figura 4. Concentraciones séricas de aspartatoaminotransferasa en niños y adultos con dengue.

Tabla 3. D istribución anual de casos confirmados de $D$ engue y niveles de transferasas en el Estado Zulia Venezuela, 1999-2001.

\begin{tabular}{|lcccccccccc|}
\hline Año & \multicolumn{4}{c}{ Fiebre por Dengue } & \multicolumn{3}{c|}{ Fiebre Hemorágica por Dengue } & \multicolumn{2}{c|}{ Total } \\
& \multicolumn{2}{c}{ Casos } & \multicolumn{2}{c}{ Trans. Elevadas } & \multicolumn{2}{c|}{ Casos } & Trans. Elevadas & & \\
& $n$ & $\%$ & $n$ & $\%$ & $n$ & $\%$ & $n$ & $\%$ & $n$ & $\%$ \\
\hline 1999 & 20 & 41,66 & 4 & 8,33 & 32 & 23,52 & 12 & 8,82 & 52 & 28,26 \\
2000 & 8 & 16,66 & 4 & 8,33 & - & - & - & - & 8 & 4,34 \\
2001 & 20 & 41,66 & 4 & 8,33 & 104 & 76,47 & 88 & 64,70 & 124 & 67,39 \\
Total & 48 & 26,08 & 12 & 25 & 136 & 73,91 & 100 & 73,52 & 184 & 100 \\
\hline
\end{tabular}

dolor abdominal localizado en hipocondrio derecho) con las concentraciones de aminotransferasas en los pacientes con dengue, se observó que $53,1 \%$ presentaron concentraciones dentro del rango normal y $46,8 \%$ estaban elevadas. Mientras que $92,8 \%$ de los pacientes sin síntomas de afectación hepática presentaron aminotransferasas elevadas y 7,1\% las tuvieron en el rango normal (Figura 5).

\section{Discusión}

Este estudio muestra un incremento significativo de las concentraciones séricas de aminotransfera- sas ALT y AST en 60,8\% de los pacientes con dengue, con valores similares a los observados en pacientes con hepatitis aguda confirmada por virus B. La elevación de ambas enzimas y la presencia de manifestaciones clínicas hepáticas sugieren una lesión hepatocelular, producto de la infección por virus dengue. Muchas veces la alteración enzimática no se relaciona con manifestaciones clínicas de disfunción hepática. Sirivichayacul y cols. ${ }^{19}$ describieron la elevación de aminotransferasas y afección hepática como manifestación infrecuente del dengue.

Marianneau y cols, destacaron las alteraciones hepáticas en pacientes con dengue como signo de mal pronóstico y característico de enfermedad 


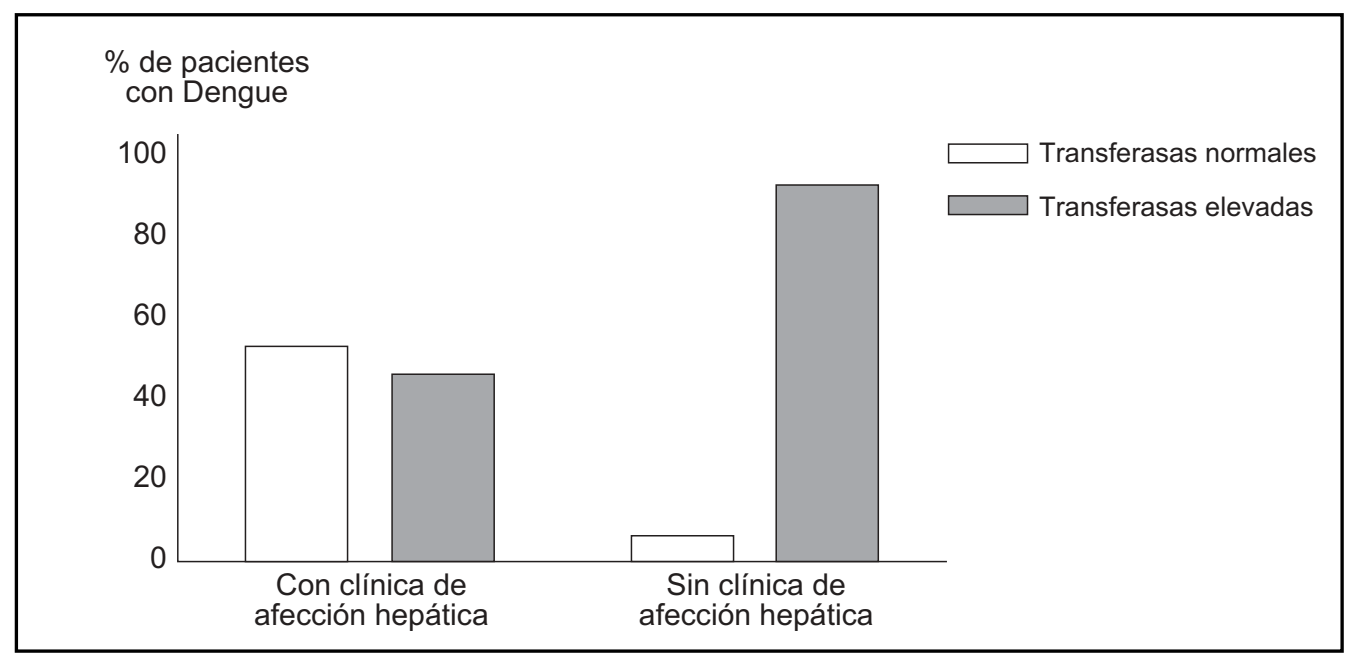

Figura 5. Manifestaciones clínicas de afectación hepática y transferasas en pacientes con Dengue.

probablemente fatal, la mayoría de las veces acompañado con encefalopatía ${ }^{20}$. Otros autores concluyeron que el virus dengue puede ocasionar injuria hepática y elevación de las enzimas hepáticas similar a lo observado en pacientes con hepatitis viral convencional ${ }^{6,21,22}$.

En el presente estudio se confirma lo reportado por Lin y cols 23 , Mohan y $\operatorname{cols}^{24}$, quienes describieron que en la hepatitis por virus dengue los niveles de AST son superiores que los de ALT; mientras que otros tipos de hepatitis virales se eleva más la ALT que AST.

Díaz y cols, realizaron un estudio en pacientes con hepatitis por virus dengue donde se observó la presencia de hepatomegalia y una ligera elevación de ambas aminotransferasas ${ }^{25}$. Al igual que los resultados aquí obtenidos, la presencia de hepatomegalia y los niveles de ambas aminotransferasas se encontraban elevadas, la AST más que la ALT. Esto pudiera estar asociado con la síntesis de esta enzima en otros tejidos, como el músculo cardíaco y estriado, implicados en la infección por virus dengue. También Deparis y $\operatorname{cols}^{26}$, durante una epidemia de dengue, encontraron un incremento en las aminotransferasas, con predominio de AST y entre los síntomas clínicos dolor abdominal y hepatomegalia.

Nguyen y $\operatorname{cols}^{27}$, Lum y cols ${ }^{28}$, Murgue y cols ${ }^{29}$, realizaron estudios en pacientes con insuficiencia hepática y que cursaban con FHD/SSD, los mismos presentaban un incremento de las aminotransferasas ALT y AST, y observaron que el nivel de AST era superior que ALT en la mayoría de los casos. Atribuyeron la gravedad del dengue a un daño multisistémico, con severos desórdenes hepáticos, con hepatomegalia asociada y hemorragias graves. En este estudio se evidenció que un número importante de casos con FHD presentaron elevación significativa de las aminotransferasas.

Souza y $\operatorname{cols}^{30}$, descubrieron una hepatitis aguda en un paciente con dengue grado III, según estos autores el análisis clínico y de laboratorio aportaron un diagnóstico diferencial con la hepatitis viral clásica.

Pancharoen y cols ${ }^{31}$, al evaluar la correlación entre cambios bioquímicos en la función hepática y severidad de infección por virus dengue en pacientes pediátricos, observaron niveles de aminotransferasas significativamente elevados en pacientes con FHD, concluyendo que los trastornos hepáticos en los pacientes con dengue es una condición común. Méndez y cols ${ }^{32}$ reportaron en niños con FHD, manifestaciones clínicas relacionadas a hepatomegalia y hepatitis. Hongsiriwon $\mathrm{S}^{33}$ reportó casos de niños de 3 a 12 meses de edad, con infección primaria que desarrollaron FHD con hepatomegalia importante. A diferencia de lo encontrado en este estudio, los pacientes 
pediátricos presentaron manifestaciones clínicas hepáticas, con valores séricos de aminotransferasas dentro de los valores referenciales.

En este estudio la población masculina y jóvenes entre 11 y 20 años fueron los más afectados, a diferencia de lo descrito por Díaz y $\operatorname{cols}^{25}$, donde la población femenina con edades comprendidas entre 15 y 34 años fueron las más afectadas. Mientras que Deparis y cols ${ }^{26}$, mostraron que la población masculina fue la más afectada, con predominio del grupo de niños de 5 a 9 años.

El DEN-3 en América se identificó primero en Nicaragua y Panamá, en 1995 la epidemia se extendió a lo largo de Centroamérica hasta México. La introducción de DEN-3 en Nicaragua en 1994 y en México en 1995 coincidió con los números crecientes de casos de FHD en esas áreas. Venezuela ha informado números crecientes de casos de dengue tipo 3 desde 1995. En el estado Zulia han aumentado los casos anuales de FHD, especialmente en el año 2001, cuando se encontró un

\section{REFERENCIAS}

1. Setiawan MN, Samsi TK, Wulur H, Sugianto D, Pool TN. Dengue: haemorrhagic fever: ultrasound as an aid to predict the severity of the disease. Pedriatric Radiol 1998; 28: 1-4.

2. MartínEz E. Dengue y dengue hemorrágico. Universidad Nacional de Quilmes. Lab. Elea. 1998; 15-8.

3. Rosen L, KHIN MM, U T. Recovery of virus from the liver of children with fatal dengue: Reflection on the pathogenesis of the disease and its possible analogy with that of yellow fever. Res Virol 1989; 140: 351-60.

4. Couvelard A, Marianneau P, Bedel C, Drouet Mt, VACHON F, Henin D et al. Report of a fatal case of dengue infection with hepatitis: demonstration of dengue antigens in hepatocytes and liver apoptosis. Human Pathol 1999; 30: 1106-10.

5. FARRERAS R. Hepatología. En: Medicina Interna. Decimocuarta Edición, 1999; 267-358.

6. Kuo CH, Tai D, Chang-Chien CS, Lan CK, Chiou SS, LuA YF. Liver biochemical test and dengue fever. Am J Trop Med Hyg 1992; 47: 265-70.

7. GuBLER DJ. Dengue and dengue haemorrhagic fever in the Americas. P R Health Sci J 1987; 6: 107-11. mayor porcentaje de pacientes con aminotransferasas elevadas ${ }^{34}$. Este hallazgo pudiera estar relacionado con una situación de hiperendemicidad aunada a la introducción del DEN-3 en Venezuela en ese año y al hecho de que algunos autores ${ }^{35}$ relacionan los genotipos de esta cepa con el desarrollo de FHD, y también a lo reportado por Souza y cols ${ }^{36}$, en el año 2004, quienes describieron cambios en los niveles de las aminotransferasas en hepatitis aguda en pacientes con DEN-3.

Es importante resaltar que en la región zuliana de Venezuela se ha observado un incremento de los casos activos de dengue que cursan con un cuadro de disfunción hepática, lo que amerita estudios que evalúen el mecanismo de afección hepática en relación al serotipo infectante.

\section{Agradecimiento}

Al Consejo de Desarrollo Científico y Humanístico de la Universidad del Zulia (CONDES) por el financiamiento otorgado CC-0789-04.

8. Guzmán MG, Kouri G, Bravo JR, Peiegrino JL. La emergencia de la fiebre hemorrágica del dengue en las Américas. Reemergencia del dengue. Rev Cubana Med Trop 1999; 51: 5-13.

9. United States Centers For Disease Control. Dengue type 3 infection. Nicaragua and Panama. MMWR 1995; 44: 21-4.

10. Guzmán MG, Vázquez S, Martínez E, Alvarez M, Rodríguez R, Kourí G. Dengue in Nicaragua 1994: reintroduction of serotype 3 in the Americas. Pan Am J Public Health 1997; 1: 193-9.

11. Briceño B, Gómez H, Argott E, Montesano R, Vázquez AL, IBáÑez $S$ et al. Potencial risk for Dengue 3 in México. Emerg Infect Dis 1996; 2: 133-5.

12. Ricco-Hesse R, Harrison LM, Salas Ra, Tovar D, NisalaK A, Ramos $C$ et al. Origins of Dengue type 2 viruses associated with increased pathogenicity in the Americas. Virology 1997; 230: 244-51.

13. Lanciotti RS, Grant J, Glubler DJ, Trent DW. Molecular evolution and epidemiology of dengue 3 viruses. J Gen Virol 1994; 75: 65-75.

14. Guzmán MG, Rosario D, Muné M, Alvarez M, Rodríguez R, Kourí G. Relaciones genéticas del virus dengue 3 aislado en la epidemia de FHD de Nicaragua. Rev Cubana Med Trop 1994; 2: 114-7. 
15. Kuno G, Gómez I, Gubler D. An ELISA procedure for the diagnosis of Dengue infections. J Virol Methods 1991; 33: 101-3.

16. Chanama S, Anantapreecha S, A-Nuegoonpipat A, SAGnasang A, Kurane I, Sawanpanyalert P. Analysis of specific IgM responses in secondary dengue virus infections: levels and positive rates in comparison with primary infections. Journal of Clinical Virology 2004; 31: 185-9.

17. Gubler DL, Kuno G, Sather E, Valez M, Oluve A. Mosquito cell cultures and specific monoclonal antibodies in surveillance for dengue virus. Am J Trop Med Hyg 1984; 33: 158-65.

18. Frankel S, Gradwohl S. Clinical laboratory methods and diagnostic. Séptima Edición. 1970; 1: 123.

19. Sirivichayakul C, Sabcharoen A, Chanthavanich $P$, Pengsaa K, Chokejindachai W, Prarinyanupharb V. Dengue infection with unusual manifestations: a case report. J Med Assoc Thai 2000; 83: 325-9.

20. Marianneau PH, StefFan A, Royer C, Drouet MT, JAECK D, KIRN A ET AL. Infection of cultures of human Kupffer cells by dengue virus: no viral progeny synthesis, but cytokine production is evident. J Virol 1999; 73: 5201-6.

21. Henchal EA, Putnak JR. The dengue viruses. Clin Microbiol Rev 1990; 3: 376-96.

22. Kalayanarooj S, Vaughn DW, Nimmannitya S, Green S, Suntayakorn S, Kunentrasai N et al. Early clinical and laboratory indicators of acute dengue illness. J Infect Dis 1997; 176: 313-21.

23. Lin YL, Lu CC, Lei HY, YeH TM, Ln YS, Chen RMY ET AL. Infection of five human liver cell lines by dengue-2 virus. J Med Virol 2000; 60: 425-31.

24. Mohan B, Patwari AK, Anand VK. Hepatic dysfunction in childhood dengue infection. J Trop Pediatr 2000; 46: 40-3.

25. Díaz S, Piñera M, Guash F. Hepatitis reactiva por virus del dengue hemorrágico. Rev Cubana Med Trop 2001; 53: 28-31.

26. Deparis X, Murgue B, Roche C, Cassar O, Chungue E. Changing clinical and biological manifestations of dengue during the dengue-2 epidemic in French Polynesia in 1996/97- description and analysis in a prospective study. Trop Med Int Health 1998; 3: 859-65.

27. Nguyen TL, Nguyen TH, Tieu NT. The impact of dengue haemorrhagic fever on liver function. Res Virol 1997; 148: 273-7.

28. Lum LC, Lam SK, George R, Devi S. Fulminant hepatitis in dengue infection. Southeast Asian J Trop Med Public Health 1993; 24: 467-71.

29. Murgue B, Deparis $X$, Chungue E, Cassar O, Roche C. Dengue: an evaluation of dengue severity in French Polynesia base don an analysis of 403 laboratory- confirmed cases. Trop Med Int Health 1999; 4: 765-73.

30. Souza LJ, Goncalves CH, Souto JT, Fereira T, Azevedo C, Neto CG et al. Hepatitis in dengue shock síndrome. Braz J Infect Dis 2002; 6: 322-7.

31. Pancharoen C, Rungsarannmont A, Thisyakorn U. Hepatic dysfunction in dengue patients whith various severity. J med Asoc Thai 2002; 85: 298301.

32. Méndez A, GonzÁlez G. Dengue haemorrhagic fever in children: ten years of clinical experience. Biomedica 2003; 23: 180-93.

33. Hongsiriwon S. Dengue hemorrhagic fever in infants. Southeast Asian J Trop Med Public Health 2002; 33: 49-55.

34. Larreal Y, Valero N, Estevíz J, Reyes I, Maldonado M, ESPINA LM ET AL. Alteraciones hepáticas en pacientes con Dengue. Invest Clin 2005; 46: 169-78.

35. República de Venezuela Ministerio de Sanidad y Asistencia Social Organización Panamericana de la Salud OPS: Normas técnicas y operativas para la prevención del dengue y el control del AEDES AEGYPTI en Venezuela. Organización Mundial de la Salud OMS, Caracas, Venezuela, 1997; 3-7.

36. Souza LJ, Alves JG, Nogueira RM, Gicovate Neto C, Bastos DA, Siqueira EW et al. Aminotransferase changes and acute hepatitis in patients with dengue fever: analysis of 1,585 cases. Braz J Infect Dis 2004; 8 156-63. 